Maike SUBE, Aachen \& Martin FRANK, Karlsruhe

\title{
Vorschlag für eine Abiturprüfungsaufgabe mit authentischem und relevantem Realitätsbezug
}

Dieser Beitrag zum Tagungsband ist eine Zusammenfassung des gleichnamigen zur Veröffentlichung eingereichten Buchkapitels im Buch „Modellierungskompetenzen - Beurteilung und Bewertung “. Neben den obengenannten Personen sind zudem Thomas Camminady und Dr. Christina Roeckerath Autoren des Buchkapitels.

Spätestens seitdem Winter (1995) die Schulung der Wahrnehmung und des Verständnisses der „Erscheinungen der Welt um uns herum“ als Bestandteil des Mathematikunterrichts gefordert hat und in den Bildungsstandards der Kultusministerkonferenz im Fach Mathematik für die Allgemeine Hochschulreife (2012) diese Forderung aufgriffen wurde, ist die Entwicklung und der Einsatz von Abiturprüfungsaufgaben mit authentischem und relevantem Realitätsbezug wichtig für die mathematische Bildung der Schülerinnen und Schüler. Einen Realitätsbezug in Abiturprüfungsaufgaben herzustellen, scheint mit Blick auf die bereits existierenden Aufgabenvorschläge möglich (u.a. KMK, 2012; IQB) - dass dieser Realitätsbezug jedoch den Anforderungen der Authentizität in Bezug auf die Situation und die Nutzung von Mathematik sowie der Relevanz entspricht, ist häufig zu bezweifeln, was Greefrath, Ludwig und Siller (2017) zum Beispiel durch Untersuchung von IQB-Aufgaben untermauerten.

Wir stellen uns der Herausforderung, tatsächlich authentische und relevante Realitätsbezüge in Abiturprüfungsaufgaben herzustellen und haben daher im Rahmen des Schülerlabors Computational And Mathematical Modeling Program (CAMMP) der RWTH Aachen und des KIT einen Vorschlag entwickelt.

\section{Der Aufgabenvorschlag}

Der Aufgabenvorschlag entstand aus einem Tagesworkshop zum Thema Datensicherheit in sozialen Netzwerken (Sube, 2016). Hintergrund ist die Präsidentschaftswahl von Donald Trump, dessen Wahlkampfteam mit der Firma Cambridge Analytica zusammenarbeitete und hier Big-Data-Analysen betrieb (u.a. Zastrow, 2016). Unter anderem dazu werden in sozialen Netzwerken sogenannten Schattenprofile von Personen genutzt, die Informationen enthalten, die die Person nicht von sich aus preisgegeben hat (Garcia, Sarigol $\&$ Schweitzer, 2014). Wie gut man solche nicht preisgegebenen Informationen - in unserem Fall das Alter bzw. die Altersgruppe eines Netzwerknutzers - vorhersagen kann, ist Fragestellung der Abiturprüfungsaufgabe. Die 
Schülerinnen und Schüler arbeiten mit einem echten Datensatz des Netzwerks Friendster. Die Aufgabe ist in das Inhaltsfeld Stochastik ein- und der Kompetenz des Modellierens zuzuordnen.

Zunächst werden die Schülerinnen und Schüler in die Thematik eingeführt. In der ersten Aufgabe vereinfachen die Schülerinnen und Schüler, indem sie anhand einer Altersverteilung Nutzer des Netzwerks Friendster, die zur Untersuchung nicht geeignet scheinen, aus dem Datensatz begründet ausschließen. Hier sehen die Schülerinnen und Schüler in der dargestellten Altersverteilung unter anderem auch Nutzer mit 0 Jahren als Altersangabe. Zur Vorhersage des Alters eines Nutzers stehen zwei Vorhersageregeln zur Verfügung, die den Schülerinnen und Schülern vorgestellt werden. Eine Regel nutzt zur Vorhersage das Alter der Freunde des Nutzers, die andere einen datenbasierten Zufall. Die Mathematisierung erfolgt durch ein Bernoulli-Experiment und durch eine binomialverteilte Zufallsvariable, was die Schülerinnen und Schüler durch ein Urnenexperiment erläutern sollen. Die „,Zufallsregel“" wird nun genauer betrachtet. Dazu werden bekannte Aufgabentypen aus bereits existierenden Abiturprüfungen, wie das Anwenden der Binomialverteilung und das Berechnen von Kenngrößen (Erwartungswert und Standardabweichung) verwendet. Zudem soll eine mögliche Approximation der Binomialverteilung durch die Normalverteilung beurteilt werden. Die „Freunde-Regel“" wird dann mit Hilfe von bedingten Häufigkeiten ebenfalls genauer betrachtet. Die beiden Regeln und eine dritte unbekannte Regel werden schließlich mit Hilfe von Trefferquoten verglichen und in ihrer Güte auch durch einen Hypothesentest - beurteilt. Das Validieren erfolgt zuerst vor allem in der Beurteilung, ob die Ergebnisse auf moderne soziale Netzwerke übertragbar sind, und dann in Bezug auf die ursprüngliche Fragestellung:

Beurteilen Sie, ob es möglich ist, das Alter von Netzwerknutzern zu bestimmen, die ihr Alter nicht explizit angegeben haben.

Schließlich sollen die Schülerinnen und Schüler eine weitere Regel zur Vorhersage des Alters herleiten.

\section{Eignung der Aufgabe}

Die Abiturprüfungsaufgabe erfüllt die Kriterien des Realitätsbezugs, der Relevanz und der Authentizität der Situation sowie der Verwendung der Mathematik. Das Thema ist Bestandteil der Lebenswelt der Schülerinnen und Schüler und sie sind vielleicht als Nutzer sozialer Netzwerke von der Analysemethode betroffen (JIM-Studie 2017). Da es sich um eine echte Situation handelt, ist sie authentisch. Die zur Lösung der Problemstellung genutzte 
Mathematik ist authentisch, da die Vorgehensweise der Forschung (vgl. Garcia, Sarigol, Schweitzer, 2014) hier lediglich exemplifiziert wurde.

Sie ist angelehnt an die bereits existierenden Formate von Abiturprüfungsaufgaben, kann in die Anforderungsbereiche der Bildungsstandards eingeordnet werden, betont jedoch das Vereinfachen und Validieren sowie die gesellschaftliche Bedeutung von Mathematik in besonderem Maße.

In ersten pilotierenden Tests wurde die Abiturprüfungsaufgabe von 105 Schülerinnen und Schülern der Qualifikationsphase 2 im Aachener Raum getestet. Die Durchführungen waren aufgrund der existierenden Rahmenbedingungen sehr divers. Dennoch können Tendenzen zur Eignung der Aufgabe abgeleitet werden. Hier werden einige Auszüge dargestellt:

Die Schülerinnen und Schüler empfanden die Aufgabenstellungen grundsätzlich als relevant und authentisch. Die meisten fanden das Thema interessant. Die Schülerinnen und Schüler waren fähig, eine angemessene, wenn auch nicht immer vollständige Lösung bei der Vereinfachung, die für sie neu war, zu erbringen. Bereits bekannte Aufgabentypen, wie die Anwendung der Binomialverteilung oder die Berechnung der Kenngrößen, wurden ebenfalls angemessen bearbeitet. Komplexere Aufgaben, wie die Begründung mittels Urnenexperiment, die Approximation oder der Hypothesentest, bereiteten den Schülerinnen und Schülern - wie erwartet - einige Probleme. Das Validieren, gerade die Betonung der Nutzung des Weltwissens in den letzten Aufgaben, war für die Schülerinnen und Schüler neu. Die Lösung war hier nicht immer vollständig, aber dennoch gut.

Grundsätzlich lässt sich also tendenziell sagen, dass bekannte Aufgabentypen für die Schülerinnen und Schülern leichter zu bearbeiten sind als die neuartigen. Dennoch können die Schülerinnen und Schüler auch diesen Aufgaben adäquat begegnen. Die Schülerinnen und Schüler merkten häufig Zeitmangel bei der Bearbeitung der Aufgaben an. Eine weitere Testung mit einem höheren Zeitkontingent ist daher zu empfehlen.

\section{Öffnung der Aufgabenstellungen}

Nach unserer Auffassung von Modellierungsaktivitäten mit Schülerinnen und Schülern ist auch die Kreativität und die Nutzung von Weltwissen Bestandteil einer Modellierung. Hier gibt es weitergehend Möglichkeiten, die Aufgabenstellungen zum Vereinfachen, zum Mathematisieren und zum Validieren zu öffnen. Als Beispiel kann eine Öffnung wie folgt lauten:

Bei der Schätzung der Altersgruppe ohne Beachtung der Freunde eines Nutzers kann man eine Trefferwahrscheinlichkeit von $51 \%$ erreichen. Ein Experiment mit einer Regel, die die Angaben der Freunde eines Nutzers nutzt, zeigte: Diese schätzt bei $81 \%$ der Nutzer die Altersgruppe richtig ein. 
Diskutieren Sie, warum die Freunde-Regel besser ist als die Einschätzung mit Hilfe des Zufalls.

Hier können die Schülerinnen und Schüler diskutieren, inwieweit konkrete Informationen über Nutzer eine Auswirkung auf die Güte der Vorhersagen haben können. Auch möglich wäre die Aufgabe:

Die Anwendung einer anderen Regel ergibt, dass $95 \%$ der Nutzer nicht zwischen 25 und 30 Jahre alt sind. Beurteilen Sie, ob dieses Ergebnis realistisch ist.

Hier wird die Frage nach der Plausibilität des Ergebnisses in den Vordergrund gestellt. Durch Weltwissen kann hier eine Einschätzung getätigt werden. Weitere Beispiele werden im Buchkapitel vorgestellt.

Mit diesem Beitrag erhoffen wir uns, die Diskussion um Abiturprüfungsaufgaben mit authentischem und relevantem Realitätsbezug weiter anzuregen. Die Testungen zeigen, dass die aktuellen Schülerinnen und Schüler diesen Weg mitgehen können. Wir ermutigen daher zur weiteren Entwicklung von Abiturprüfungsaufgaben mit authentischem und relevantem Realitätsbezug.

\section{Literatur}

Garcia, D., Sarigol, E. \& Schweitzer, F. (22.09.2014). Online Privacy as a Collective Phenomenon. COSN'14, Dublin. Abgerufen am 06.06.2016, von http://arxiv.org/pdf/1409.6197v1.pdf.

Greefrath, G. Siller, H.-S. \& Ludwig, M. (2017). Modelling Problems in German Grammar School Leaving Examinations (Abitur) - Theory and Practice. Proceedings of the Tenth Congress of the European Society for Research in Mathematics Education (CERME 10), 829-836, Dublin, Ireland.

Institut zur Qualitätsentwicklung im Bildungswesen (IQB). Abgerufen am 03.04.2018, von https://www.iqb.hu-berlin.de/abitur/sammlung und www.iqb.hu-berlin.de/bista/abi

Kultusministerkonferenz der Länder in der Bundesrepublik Deutschland (KMK). (2015) Bildungsstandards im Fach Mathematik für die Allgemeine Hochschulreife. Wolters, Kluver: München, 2015.

Medienpädagogischer Forschungsverbund Südwest. (2017). JIM 2017 Jugend, Information, (Multi-) Media - Basisstudie zum Medienumgang 12- bis 19-Jähriger in Deutschland. Stuttgart, November 2017. Abgerufen am 18.12.2017, von www.miz.org/downloads/dokumente/908/2017_JIM-Studie_mpfs.pdf

Sube, M. (2016) Wie sicher ist meine Privatsphäre in sozialen Netzwerken? ...und was hat das mit Mathe zu tun?, Masterarbeit, RWTH Aachen. Abgerufen am 03.04.2018, von https://blog.rwth-aachen.de/cammp/files/2016/10/thesis- soziale-netzwerke.pdf

Winter, H. (1995). Mathematikunterricht und Allgemeinbildung. (1995) Mitteilungen der Gesellschaft für Didaktik der Mathematik, 61, 37-46. Abgerufen am 06.06.2016, von http://geosoft.ch/fachdidaktik/fd3/Winter.pdf

Zastrow, V. (12.12.2016). Wie Trump gewann. Frankfurter Allgemeine. Abgerufen am 20.12.2016, von http://www.faz.net/aktuell/politik/trumps-praesidentschaft/wie-der-wahlsieg-vondonald-trump-mit-big-data-gelang-14568868.html?printPagedArticle=true \#pageIndex_2 